\title{
Guiding of Intense Laser Beams in Highly Ionized Plasma Columns Generated by a Fast Capillary Discharge
}

\author{
B. M. Luther, ${ }^{*}$ Y. Wang, M. C. Marconi, J. L. A. Chilla, M. A. Larotonda, and J. J. Rocca ${ }^{\dagger}$ \\ NSF ERC for Extreme Ultraviolet Science and Technology, and Electrical and Computer Engineering Department, \\ Colorado State University, Fort Collins, Colorado 80523, USA
}

(Received 22 September 2003; published 10 June 2004)

\begin{abstract}
We have demonstrated the guiding of laser pulses with peak intensities up to $2.2 \times 10^{17} \mathrm{~W} / \mathrm{cm}^{2}$ in a $5.5 \mathrm{~cm}$ long plasma column containing highly charged Ar ions generated by a fast capillary discharge. A rapid discharge-driven hydrodynamic compression guides progressively lower order modes through a plasma with increasing density and degree of ionization, until the guide collapses on axis. The lowest order mode (FWHM $\sim 50 \mu \mathrm{m}$ ) is guided with $75 \%$ transmission efficiency shortly before the plasma reaches the conditions for lasing in Ne-like Ar. The subsequent rapid plasma expansion forms a significantly leakier and more absorbent guide.
\end{abstract}

The guiding of intense laser beams in plasmas has attracted significant attention [1-9]. This is motivated by the need for extending the interaction length between intense laser pulses and plasmas beyond the limitations set by diffractive defocusing and ionization-induced refraction for important applications such as the generation of intense coherent soft x-ray radiation [9-12] and wakefield accelerators $[13,14]$. Preformed index waveguides, plasma channels with an electron density minima on axis, were first demonstrated using the hydrodynamic expansion of a cylindrical plasma following a laser-produced spark $[3,4,9]$. More recently, several approaches based on electrical discharges have been studied, including plasmas created by discharge ablation of the walls of a microcapillary [5], a discharge through a hydrogen filled microcapillary [6], and the plasma implosion in Z-pinch discharges in helium and methane [7,8].

An application of considerable interest for plasma waveguides is the longitudinal excitation of soft $\mathrm{x}$-ray lasers that can potentially result in saturated amplifiers with reduced laser pump energy and increased efficiency [9-12]. Lasing at $60.8 \mathrm{~nm}$ by collisional electron excitation of S VIII [10], collisional recombination in Li III at $13.5 \mathrm{~nm}$ [11], and optical-field ionization driven collisional excitation in Pd-like Xe at $41.8 \mathrm{~nm}$ [12] has been reported in laser-driven plasma channels created in wall ablated $[10,11]$ and gas filled [12] microcapillaries excited by relatively slow discharge current pulses. Particularly promising is the development of transient collisional lasers using Ne-like or Ni-like ions $[10,15,16]$.

Herein we report the characterization of multiply ionized plasma waveguides created by a fast Ar capillary discharge of the type used to develop discharge-pumped collisional soft $\mathrm{x}$-ray lasers [17-19] and the demonstration of the guiding of laser pulses with peak intensity up to $2.2 \times 10^{17} \mathrm{~W} \mathrm{~cm}^{-2}$. In contrast to slow capillary discharges these discharges can reach the Ne-like or Ni-like stage of ionization for several atoms of interest [18]. A rapid compression of the plasma column results from the strong current-induced $J \times B$ force [17]. A shock wave that originates in the vicinity of the capillary wall propagates towards the axis forming a plasma waveguide of continuously decreasing diameter, and increasing density and degree of ionization, until it collapses on axis. The nearly $100 \times$ volumetric plasma compression achieved with these fast discharges allows the generation of dense plasma columns using initial gas pressures of $<1$ Torr. This low initial gas pressure constitutes a potential advantage for the implementation of soft $\mathrm{x}$-ray laser amplifiers, as it reduces the problem of absorption of the amplified radiation by neutral gas at the ends of the plasma column.

The experiments made use of a fast desktop Ar capillary discharge and a Ti:Sa short pulse laser system containing up to three stages of amplification. Most of the experiments used $\sim 2.5 \mathrm{ps}$ pulses, except for the demonstration of high intensity beam guiding that was done using $\sim 100 \mathrm{fs}$ pulses. The discharge utilized $3.2 \mathrm{~mm}$ diameter, 5.5 to $11 \mathrm{~cm}$ long $\mathrm{Al}_{2} \mathrm{O}_{3}$ capillaries filled with 180 to 250 mTorr of Ar. The gas was continuously flown and a $600 \mu \mathrm{m}$ diameter hole in one of the electrodes allowed the injection of the Ti:Sa laser while maintaining a pressure on the order of $1 \times 10^{-4}$ Torr outside the discharge channel using differential pumping. The discharge current pulses had a peak amplitude of 15 to $21 \mathrm{kA}$ and a half-period of $\sim 120 \mathrm{~ns}$. As shown in Fig. 1 for a $11 \mathrm{~cm}$ long capillary the current pulse is nonsinusoidal and presents a kink at the time of the pinch that results from the increase in inductance caused by the plasma column compression. Synchronization between the capillary discharge and the laser pulse injection was obtained by triggering a pressurized spark gap with a focused $\sim 1 \mathrm{~mJ}, 800 \mathrm{~nm}$, laser pulse compressed to $\sim 50 \mathrm{fs}$. For most of these experiments the jitter was on the order of a few ns, and subns jitter is possible [19]. The time of the pinch is dependent on the capillary length, pressure, and 


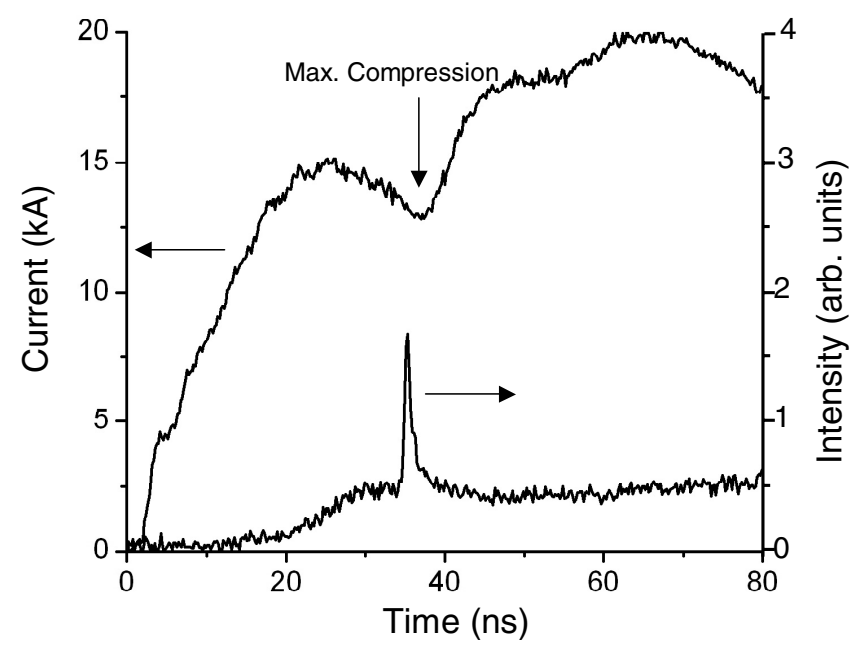

FIG. 1. Discharge current pulse for $11 \mathrm{~cm}$ long capillary (upper trace), and vacuum photodiode signal (lower trace) showing lasing at $46.9 \mathrm{~nm}$ in Ne-like Ar $1.5 \pm 0.3 \mathrm{~ns}$ before the time of maximum compression. The Ar pressure was 189 mTorr.

current. The plasma compression is accompanied by an increase in the degree of ionization, reaching $Z=8$ shortly before the pinch, as corroborated during these experiments by the observation of lasing at $46.9 \mathrm{~nm}$ in Ne-like Ar. Maximum amplification occurred $1.5 \pm$ $0.3 \mathrm{~ns}$ before the kink in the current pulse (Fig. 1).

Figure 2 shows a sequence of interferometrically measured electron density profiles depicting the early stage of the plasma waveguide formation. The data were obtained probing the plasma with subps laser pulses of $\lambda=$ $267 \mathrm{~nm}$, at the time when the column diameter is large, the plasma density is in the range of $(1-5) \times 10^{17} \mathrm{~cm}^{-3}$, and refraction of the probe pulses is negligible. The data show the evolution of a highly symmetric annular plasma shell with density minimum on the center that constitutes

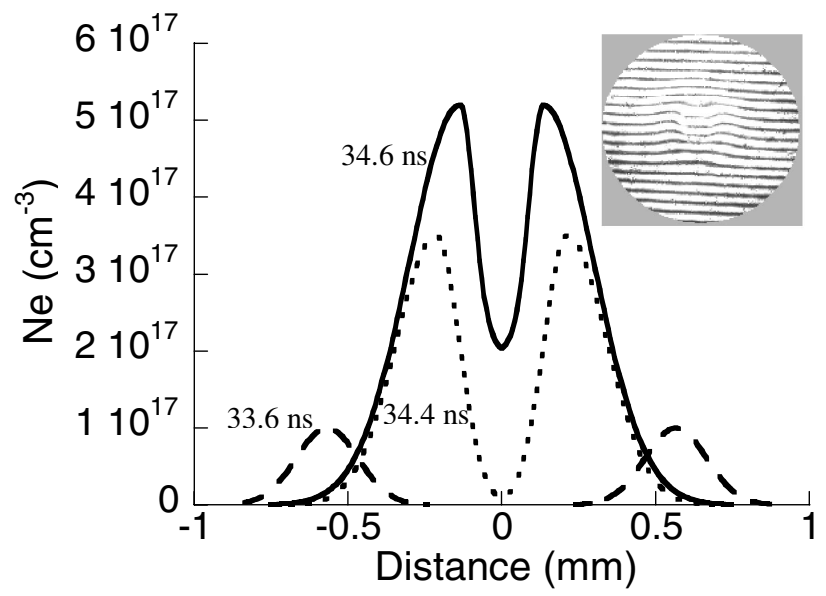

FIG. 2. Electron density profiles obtained from interferograms corresponding to the early stage of guide formation in a $1.4 \mathrm{~cm}$ long capillary. The times indicated are measured with respect to the beginning of the current pulse. The inset shows the interferogram corresponding to the $34.4 \mathrm{~ns}$ profile. a waveguide of continuously decreasing diameter and increasing density.

The guiding properties of the plasma columns were investigated using near- and far-field imaging, transmission measurements, and modeling. For these measurements a capillary length of $5.5 \mathrm{~cm}$ was chosen after experimentally verifying that this length does not obstruct the exit beam even at the time of the pinch when strong refraction occurs. Pulse energies between 1 and $100 \mathrm{~mJ}$ were focused at the entrance of the capillary to a measured spot of $\omega_{0}=30 \mu \mathrm{m}$ using a combination of $f=7 \mathrm{~m}$ and $f=1 \mathrm{~m}$ spherical lenses $\left(Z_{r} \sim 3.5 \mathrm{~mm}\right.$, $F \# \sim 57)$. Figure 3 shows the progression of the output mode intensity distribution as a function of time with respect to the initiation of the current pulse. For times less than $30 \mathrm{~ns}$ the plasma density is low and the peak of the electron density is far from the axis (i.e., the waveguide has a large diameter), resulting in no guiding of the beam. When the plasma column compresses to $\sim 800 \mu \mathrm{m}$ in diameter, the wings of the pulse begin to experience a phase shift that results in output modes with concentric circles as shown in Fig. 3(a). As the column continues to compress, a variety of lower order modes, such as that illustrated in Fig. 3(b), are seen. At this stage, modeling shows unstable periodic refocusing occurs inside the capillary. As the column compresses further, only the lowest order mode is guided. Figure 3(c) shows an approximately Gaussian exit mode with a FWHM of $\sim 50 \mu \mathrm{m}$. Subsequently, the guide is lost as the plasma pinches and the electron density profile becomes convex, resulting in strong beam refraction [Fig. 3(d)]. The development of a second guiding phase was observed to take place during the first few ns of the expansion that follows the pinch, as seen in Fig. 3(e). However, as discussed below, intentionally saturated near- and far-field beam patterns and their comparison with beam propagation simulations reveal the postpinch guides are significantly leakier.

Figures 4(a) and 4(b) show exit mode patterns for the prepinch waveguide imaged at 0 and $7.5 \mathrm{~cm}$ from the exit of the guide, respectively. The corresponding beam propagation simulation is displayed on the upper part of the figure. The modeling was accomplished computing the Kirchhoff-Fresnel integral using a fast-Fourier transform method [20] using the radially dependent phase delay determined from the interferometrically measured density profile. The exit mode patterns and the simulation for a guide with a channel depth of $\Delta \mathrm{Ne}=3 \times 10^{17} \mathrm{~cm}^{-3}$ at $r=120 \mu \mathrm{m}$ show the prepinch guide channels the fundamental mode with little leakage. In contrast, the near-field exit pattern for the postpinch guide [Fig. 4(c)] shows the central guided mode is surrounded by a lower intensity region, which the accompanying beam propagation simulation illustrates is the result of radiation leaking at a constant angle along the length of the waveguide. Farther from the exit plane, these leaky rays continue to open, generating a diverging cone that gives 

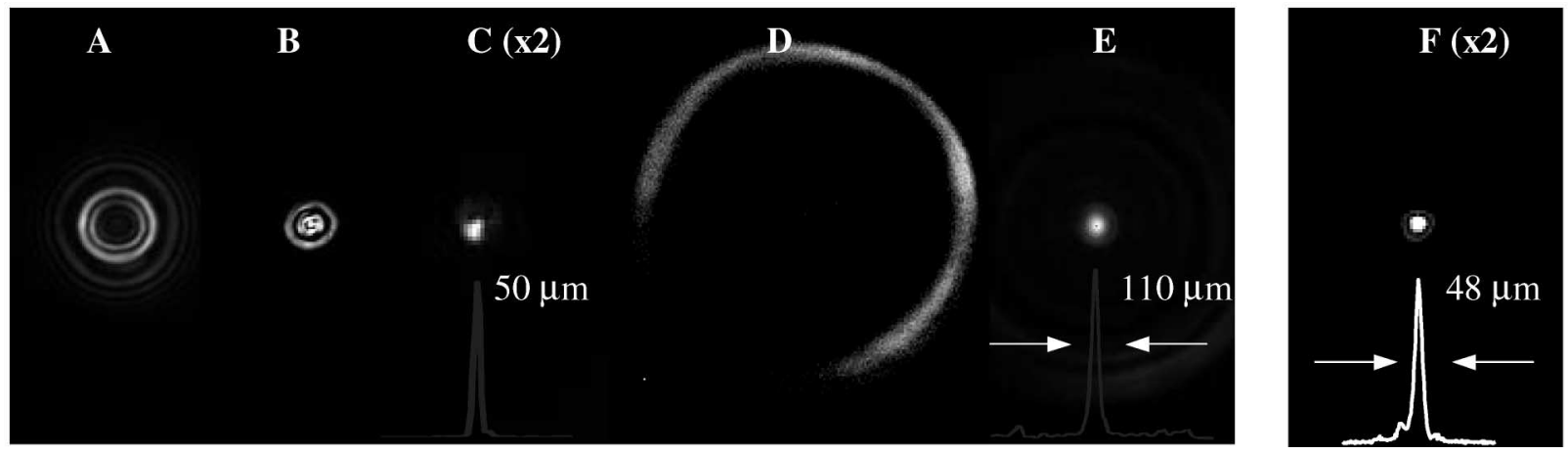

FIG. 3. (a)-(e) Sequence of images showing the evolution of the exit mode profile as a function of time (see times in Fig. 5). The capillary length was $5.5 \mathrm{~cm}$ and the laser pulse intensity $\sim 1 \times 10^{14} \mathrm{~W} \mathrm{~cm}^{-2}$. (f) Exit intensity profile for a laser pulse with a peak input intensity of $2.2 \times 10^{17} \mathrm{~W} \mathrm{~cm}^{-2}$ injected $1.8 \mathrm{~ns}$ before the pinch. The peak current was $20 \mathrm{kA}$. (c) and (f) are magnified by $2 \times$ with respect to the other images.

origin to rings of nearly constant thickness observed in Fig. 4(d). These postpinch mode patterns are consistent with an electron density profile with a very shallow channel depth of $\Delta \mathrm{Ne}=5 \times 10^{16} \mathrm{~cm}^{-3}$ and $r \sim 50 \mu \mathrm{m}$ on top of a Gaussian profile.
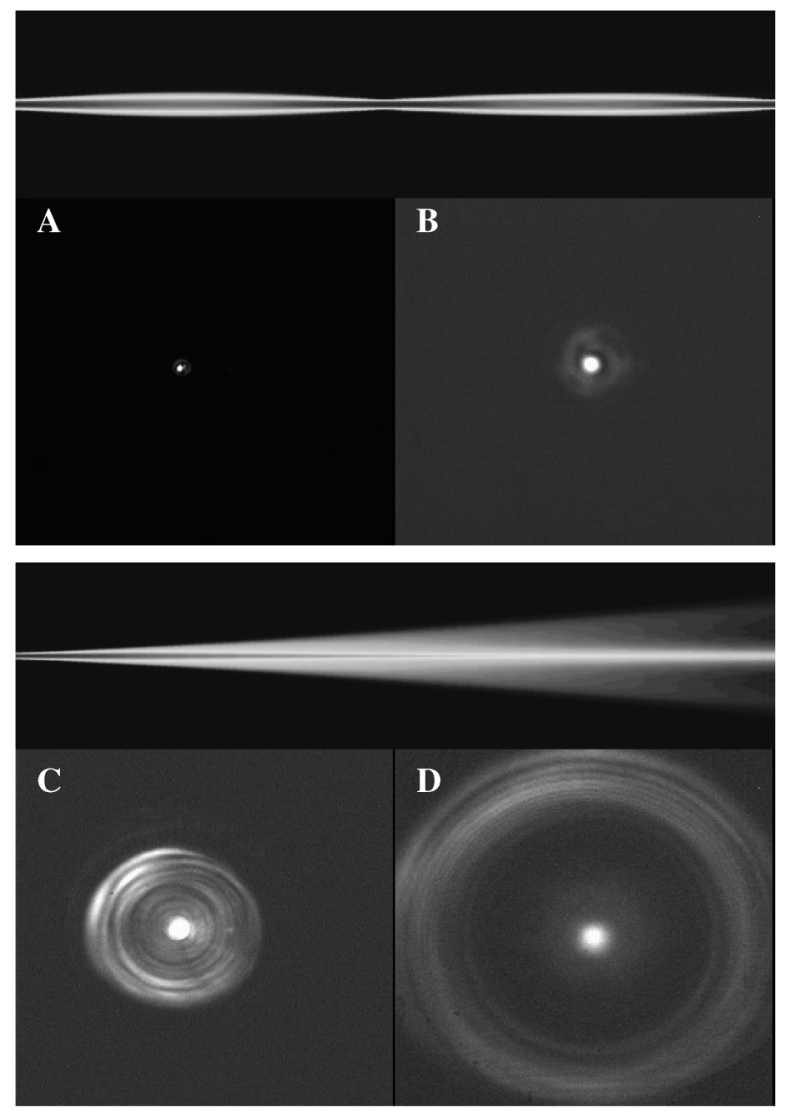

FIG. 4. Near-field (a),(c) and far-field (b),(d) images of the exit beam shortly before (top) and after (bottom) the time of maximum compression. The images were intentionally saturated to show the leaky nature of the late guides. The corresponding simulated axial beam propagation in a $5.5 \mathrm{~cm}$ capillary is shown for each case.
Channels composed of Ar VIII or Ar IX ions are immune to tunnel ionization for intensities $<3 \times$ $10^{16} \mathrm{~W} \mathrm{~cm}^{-2}$ and $<1.5 \times 10^{18} \mathrm{~W} \mathrm{~cm}^{-2}$, respectively [21]. We verified that the prepinch matched guides are maintained at high intensities by injecting pulses with a measured average energy of $500 \mathrm{~mJ}$ and pulse duration of $112 \mathrm{fs}$, corresponding to a peak input intensity of $2.2 \times$ $10^{17} \mathrm{~W} \mathrm{~cm}^{-2}$. The exit intensity distribution patterns were observed to be similar to those recorded for the lower intensity beams, with slightly larger pedestal, as illustrated in Fig. 3(f).

Transmission experiments were performed by placing a Joule meter at the exit of the capillary, taking care the entire exit beam is always captured. The amount of light transmitted can be affected by several factors including coupling losses, continuous leakage of light along the entire guide length due to the finite thickness of the waveguide wall [4], and inverse bremsstrahlung absorption. In the far field, coupling losses and leakage show up as a diverging ring around the guided mode. This allows the use of an iris for the separation of the energy of the guided mode (iris closed) from the total energy transmitted (iris open). The transmission curves in Fig. 5 are the result of averaging multiple shots at each time step relative to the beginning of the current pulse. The dashed lines labeled (a)-(e) in Fig. 5 represent the times of the images shown in Fig. 3. The difference between the measured total transmitted energy (solid curve, iris open) and guided beam energy (dashed curve, iris closed) is indicative of the amount of light lost due to poor coupling and leakage from the guide, or by refraction near the time of the pinch when the waveguide is lost. For early times, when the electron density is less than $1 \times$ $10^{18} \mathrm{~cm}^{-3}$, the total transmission is greater than $90 \%$. As the column compresses towards the pinch and guides increasingly lower order modes, the guided transmission is seen to drop slightly from about $85 \%$ to $75 \%$ for times $5(a)-5(c)$. Since at the same time the total amount of transmitted light is observed to remain relatively unchanged, the drop in guided light transmission can be 


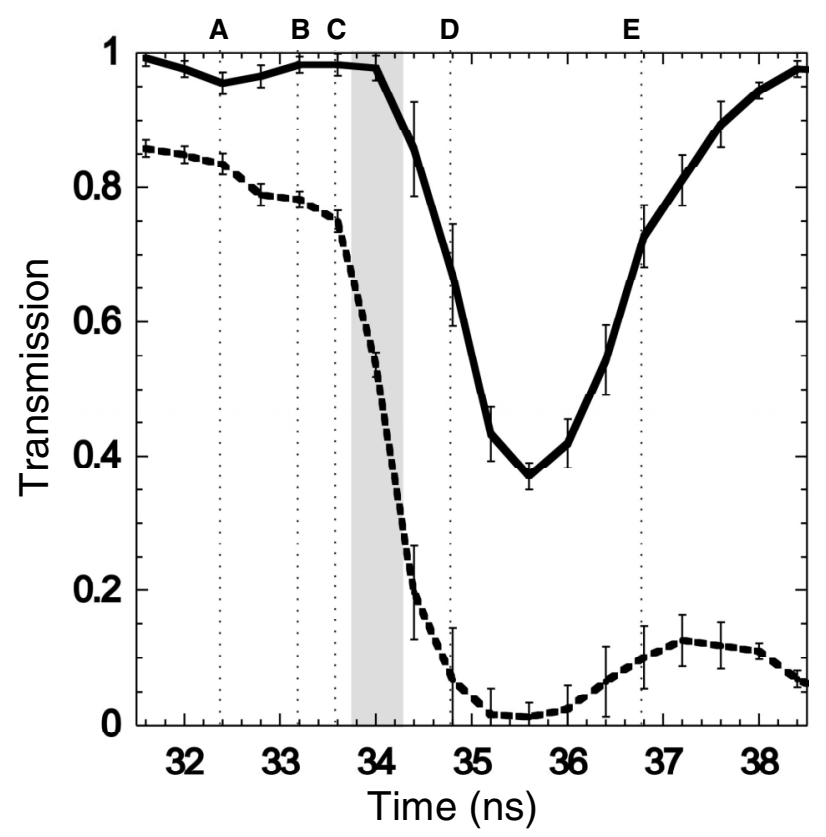

FIG. 5. Total transmission (solid line, iris open) and guided transmission (dashed line, iris closed) of $5 \mathrm{~mJ}, 2.5 \mathrm{ps} \mathrm{Ti}: \mathrm{Sa}$ laser pulses through a $5.5 \mathrm{~cm}$ long plasma column as a function of time relative to the beginning of the current pulse. Dotted lines (a)-(e) indicate times of the exit mode images in Fig. 3. The shaded area indicates the times at which the discharge driven plasma column achieves the conditions for amplification at $46.9 \mathrm{~nm}$.

attributed to an increase in uncoupled light due to the decreasing size of the guide and associated increased sensitivity to shot to shot beam walk. Guiding of the lowest order mode 5(c) occurs a fraction of a ns before the time of lasing. As seen in the dashed curve, after time 5 (c) the amount of guided light rapidly drops. This can be attributed to inverse bremsstrahlung absorption as the density increases abruptly, to decreased coupling as the guide shrinks below the $60 \mu \mathrm{m}$ diameter of the input beam, and to increased leakage as the electron density difference $\Delta \mathrm{Ne}$ decreases. As the time of the pinch is approached, the guide collapses, the electron density profile becomes convex with a maximum on axis, and the amount of guided light decreases to zero. This is consistent with the strong refraction in Fig. 3(d). The electron density rapidly increases to a maximum and the total transmission decreases to $\sim 40 \%$ due to inverse bremsstrahlung absorption. The transmission rises again shortly after the pinch, as the plasma begins to expand and the electron density decreases [Fig. 3(e)]. At the time of this late guide, occurring $\sim 1.5 \mathrm{~ns}$ after the pinch, the guided transmission is $10 \%-15 \%$. Modeling shows at this time the column remains highly ionized with $Z \sim 8$, and the solid curve in Fig. 5 indicates an absorption of about $25 \%$. This absorption, which is larger than that observed at the time of the prepinch waveguides, can be estimated to correspond to an electron density of $\sim(2-3) \times 10^{18} \mathrm{~cm}^{-3}$ assuming an electron temperature of $50 \mathrm{eV}$. An increase of the peak current would generate stronger shock waves and plasma channels with larger axial and wall densities.

In conclusion, we have characterized two distinct regimes of optical guiding in highly ionized argon plasma channels generated by a fast capillary discharge, and demonstrated the ability to propagate pulses with peak intensities up to $2.2 \times 10^{17} \mathrm{~W} \mathrm{~cm}^{-2}$. The capillaries were fired repetitively for several thousand shots without degradation. The high degree of ionization of these plasma guides and their scalability to longer lengths make them attractive for applications such as the development of compact and efficient collisional transient soft $\mathrm{x}$-ray lasers.

We gratefully acknowledge the supported of NSF Grant No. ECS-9977677, the NSF ERC for Extreme Ultraviolet Science and Technology, and the W. M. Keck Foundation.

*Electronic address: bml@lamar.colostate.edu

†Electronic address: rocca@engr.colostate.edu

[1] A. Borisov et al., Phys. Rev. Lett. 68, 2309 (1992).

[2] P. Sprangle, E. Esarey, J. Krall, and G. Joyce, Phys. Rev. Lett. 69, 2200 (1992).

[3] C. G. Durfee III and H. M. Milchberg, Phys. Rev. Lett. 71, 2409 (1993)

[4] C. G. Durfee III, J. Lynch, and H. M. Milchberg, Phys. Rev. E 51, 2368 (1995).

[5] Y. Ehrlich et al., Phys. Rev. Lett. 77, 4186 (1996).

[6] A. Butler, D. J. Spence, and S. M. Hooker, Phys. Rev. Lett. 89, 185003 (2002).

[7] T. Hosokai et al., Opt. Lett. 25, 10 (2000).

[8] C. Fauser and H. Langhoff, Appl. Phys. B 71, 607 (2000).

[9] H. M. Milchberg, C. G. Durfee III, and J. Lynch, J. Opt. Soc. Am. B 12, 731 (1995).

[10] K. A. Janulewicz et al., Phys. Rev. A 63, 033803 (2001).

[11] D. Korobkin, C. H. Nam, S. Suckewer, and A. Goltsov, Phys. Rev. Lett. 77, 5206 (1996).

[12] A. Butler et al., Phys. Rev. Lett. 91, 205001 (2003).

[13] T. Tajima and J. M. Dawson, Phys. Rev. Lett. 43, 267 (1979).

[14] P. Sprangle, E. Esarey, A. Ting, and G. Joyce, Appl. Phys. Lett. 53, 2146 (1988); P.V. Nickles et al., Phys. Rev. Lett. 78, 2748 (1997).

[15] J. Dunn et al., Phys. Rev. Lett. 84, 4834 (2000).

[16] J. J. Rocca et al., Phys. Rev. Lett. 73, 2192 (1994).

[17] J. J. Rocca, D. P. Clark, J. L. A. Chilla, and V. N. Shlyaptsev, Phys. Rev. Lett. 77, 1476 (1996).

[18] C. D. Macchietto, B. R. Benware, and J. J. Rocca, Opt. Lett. 24, 1115 (1999).

[19] B. Luther, L. Furfaro, A. Klix, and J. J. Rocca, Appl. Phys. Lett. 79, 3248 (2001).

[20] A. Siegman, Lasers (University Science Press, Mill Valley, CA, 1986), Chap. 20.

[21] B. M. Penetrante and J. N. Bardsley, Phys. Rev. A 43, 3100 (1991). 